\title{
SUBSPACE CONCENTRATION OF DUAL CURVATURE MEASURES OF SYMMETRIC CONVEX BODIES
}

\author{
KÁROLY J. BÖRÖCZKY, MARTIN HENK, AND HANNES POLLEHN
}

ABSTRACT. We prove a tight subspace concentration inequality for the dual curvature measures of a symmetric convex body.

\section{INTRODUCTION}

Let $\mathcal{K}^{n}$ denote the set of convex bodies in $\mathbb{R}^{n}$, i.e., all convex and compact subsets $K$ having a non-empty interior. The set of convex bodies having the origin as an interior point and the set of origin-symmetric convex bodies, i.e., those sets which satisfy $K=-K$ are denoted by $\mathcal{K}_{o}^{n}$ and $\mathcal{K}_{e}^{n}$ respectively. For $\boldsymbol{x}, \boldsymbol{y} \in \mathbb{R}^{n}$, let $\langle\boldsymbol{x}, \boldsymbol{y}\rangle$ denote the standard inner product and $|\boldsymbol{x}|=\sqrt{\langle\boldsymbol{x}, \boldsymbol{x}\rangle}$ the Euclidean norm. We write $B_{n}$ for the $n$-dimensional Euclidean unit ball, i.e., $B_{n}=\left\{\boldsymbol{x} \in \mathbb{R}^{n}:|\boldsymbol{x}| \leq 1\right\}$ and $S^{n-1}$ for its boundary. The $k$-dimensional Hausdorff-measure will be denoted by $\mathcal{H}^{k}(\cdot)$ and instead of $\mathcal{H}^{n}(\cdot)$ we will also write $\operatorname{vol}(\cdot)$ for the $n$-dimensional volume.

At the heart of the Brunn-Minkowski theory is the study of the volume functional with respect to the Minkowski addition of convex bodies. This leads to the theory of mixed volumes and, in particular, to the quermassintegrals $\mathrm{W}_{i}(K)$ of a convex body $K \in \mathcal{K}^{n}$. The latter may be defined via the classical Steiner formula, expressing the volume of the Minkowski sum of $K$ and $\lambda B_{n}$, i.e., the volume of the parallel body of $K$ at distance $\lambda$ as a polynomial in $\lambda$ (cf., e.g., [40, Sect. 4.2])

$$
\operatorname{vol}\left(K+\lambda B_{n}\right)=\sum_{i=0}^{n} \lambda^{i}\left(\begin{array}{l}
n \\
i
\end{array}\right) \mathrm{W}_{i}(K) .
$$

A more direct geometric interpretation is given by Kubota's integral formula (cf., e.g., [40, Subsect. 5.3.2]), showing that they are - up to some constants - the means of the volumes of projections

$$
\mathrm{W}_{n-i}(K)=\frac{\operatorname{vol}\left(B_{n}\right)}{\operatorname{vol}_{i}\left(B_{i}\right)} \int_{G(n, i)} \operatorname{vol}_{i}(K \mid L) \mathrm{d} L, \quad i=1, \ldots, n,
$$

where $\operatorname{vol}_{i}(\cdot)$ denotes the $i$-dimensional volume, integration is taken with respect to the rotation-invariant probability measure on the Grassmannian $G(n, i)$ of all $i$-dimensional linear subspaces and $K \mid L$ denotes the image of the orthogonal projection onto $L$.

1991 Mathematics Subject Classification. 52A40, 52A38.

Key words and phrases. dual curvature measure, cone-volume measure, surface area measure, integral curvature, $L_{p}$-Minkowski Problem, logarithmic Minkowski problem, dual Brunn-Minkowski theory.

First named author is supported by NKFIH 116451 and 109789. 
A local version of the Steiner formula above leads to two important series of geometric measures, the area measures $\mathrm{S}_{i}(K, \cdot)$ and the curvature measures $\mathrm{C}_{i}(K, \cdot), i=0, \ldots, n-1$, of a convex body $K$. Here we will only briefly describe the area measures since with respect to characterization problems of geometric measures they form the "primal" counterpart to the dual curvature measures we are interested in.

To this end, we denote for $\omega \subseteq S^{n-1}$ by $\nu_{K}^{-1}(\omega) \subseteq \partial K$ the set of all boundary points of $K$ having an outer unit normal in $\omega$. We use the notation $\nu_{K}^{-1}$ in order to indicate that for smooth convex bodies $K$ it is the inverse of the Gauss map assigning to a boundary point of $K$ its unique outer unit normal. Moreover, for $\boldsymbol{x} \in \mathbb{R}^{n}$ let $r_{K}(\boldsymbol{x}) \in K$ be the point in $K$ closest to $\boldsymbol{x}$. Then for a Borel set $\omega \subseteq S^{n-1}$ and $\lambda>0$ we consider the local parallel body

$$
B_{K}(\lambda, \omega)=\left\{\boldsymbol{x} \in \mathbb{R}^{n}: 0<\left|\boldsymbol{x}-r_{K}(\boldsymbol{x})\right| \leq \lambda \text { and } r_{K}(\boldsymbol{x}) \in \nu_{K}^{-1}(\omega)\right\} .
$$

The local Steiner formula is now a polynomial in $\lambda$ whose coefficients are (up to constants depending on $i, n$ ) the area measures (cf., e.g., [40, Sect. 4.2])

$$
\operatorname{vol}\left(B_{K}(\lambda, \omega)\right)=\frac{1}{n} \sum_{i=1}^{n} \lambda^{i}\left(\begin{array}{l}
n \\
i
\end{array}\right) \mathrm{S}_{n-i}(K, \omega) .
$$

$\mathrm{S}_{n-1}(K, \cdot)$ is also known as the surface area measure of $K$. The area measures may also be regarded as the (right hand side) differentials of the quermassintegrals, since for $L \in \mathcal{K}^{n}$

$$
\lim _{\epsilon \downarrow 0} \frac{\mathrm{W}_{n-1-i}(K+\epsilon L)-\mathrm{W}_{n-1-i}(K)}{\epsilon}=\int_{S^{n-1}} h_{L}(\boldsymbol{u}) \mathrm{dS}_{i}(K, \boldsymbol{u}) .
$$

Here $h_{L}(\cdot)$ denotes the support function of $L$ (cf. Section 2). Also observe that $\mathrm{S}_{i}\left(K, S^{n-1}\right)=n \mathrm{~W}_{n-i}(K), i=0, \ldots, n-1$.

To characterize the area measures $\mathrm{S}_{i}(K, \cdot), i \in\{1, \ldots, n-1\}$, among the finite Borel measures on the sphere is a cornerstone of the Brunn-Minkowski theory. Today this problem is known as the Minkowski-Christoffel problem, since for $i=n-1$ it is the classical Minkowski problem and for $i=1$ it is the Christoffel problem. We refer to [40, Chapter 8] for more information and references.

There are two far-reaching extensions of the classical Brunn-Minkowski theory, both arising basically by replacing the classical Minkowski-addition by another additive operation (cf. [17]). The first one is the $L_{p}$ addition introduced by Firey (see, e.g., [12]) which leads to the rich and emerging $L_{p}$-Brunn-Minkowski theory for which we refer to [40, Sect. 9.1, 9.2]).

The second one, introduced by Lutwak [29], is based on the radial addition $\widetilde{+}$ where $\boldsymbol{x} \widetilde{+} \boldsymbol{y}=\boldsymbol{x}+\boldsymbol{y}$ if $\boldsymbol{x}, \boldsymbol{y}$ are linearly dependent and $\mathbf{0}$ otherwise. Considering the volume of radial additions leads to the dual Brunn-Minkowski theory (cf. [40, Sect. 9.3]) with dual mixed volumes, and, in particular, also with dual quermassintegrals $\widetilde{\mathrm{W}}_{i}(K)$ arising via a dual Steiner formula (cf. (1.1))

$$
\operatorname{vol}\left(K \widetilde{+} \lambda B_{n}\right)=\sum_{i=0}^{n} \lambda^{i}\left(\begin{array}{c}
n \\
i
\end{array}\right) \widetilde{\mathrm{W}}_{i}(K)
$$


In general the radial addition of two convex sets is not a convex set, but the radial addition of two star bodies is again a star body. This is one of the features of the dual Brunn-Minkowski theory which makes it so useful. The celebrated solution of the Busemann-Petty problem is amongst the recent successes of the dual Brunn-Minkowski theory, cf. [13, 18, 44], and it also has connections and applications to integral geometry, Minkowski geometry, and the local theory of Banach spaces.

In analogy to Kubota's formula $(1.2)$ the dual quermassintegrals $\widetilde{\mathrm{W}}_{i}(K)$ admit the following integral geometric representation as the means of the volumes of sections (cf. [40, Sect. 9.3])

$$
\widetilde{\mathrm{W}}_{n-i}(K)=\frac{\operatorname{vol}\left(B_{n}\right)}{\operatorname{vol}_{i}\left(B_{i}\right)} \int_{G(n, i)} \operatorname{vol}_{i}(K \cap L) \mathrm{d} L, \quad i=1, \ldots, n .
$$

There are many more "dualities" between the classical and dual theory, but there were no dual geometric measures corresponding to the area and curvature measures. This missing link was recently established in the groundbreaking paper [25] by Huang, Lutwak, Yang and Zhang. Let $\rho_{K}$ be the radial function (see Section 2 for the definition) of a convex body $K \in \mathcal{K}_{o}^{n}$. Analogous to (1.3) we consider for a Borel set $\eta \subseteq S^{n-1}$ and $\lambda>0$ the set

$$
\begin{aligned}
& \widetilde{A}_{K}(\lambda, \eta)= \\
& \quad K \cup\left\{\boldsymbol{x} \in \mathbb{R}^{n} \backslash K: 0 \leq\left|\boldsymbol{x}-\rho_{K}(\boldsymbol{x}) \boldsymbol{x}\right| \leq \lambda \text { and } \rho_{K}(\boldsymbol{x}) \boldsymbol{x} \in \nu_{K}^{-1}(\eta)\right\} .
\end{aligned}
$$

Then there also exists a local Steiner type formula of these local dual parallel sets [25, Theorem 3.1] (cf. (1.4))

$$
\operatorname{vol}\left(\widetilde{A}_{K}(\lambda, \eta)\right)=\sum_{i=0}^{n}\left(\begin{array}{c}
n \\
i
\end{array}\right) \lambda^{i} \widetilde{\mathrm{C}}_{n-i}(K, \eta) .
$$

$\widetilde{\mathrm{C}}_{i}(K, \eta)$ is called the ith dual curvature measure and they are the counterparts to the curvature measures $\mathrm{C}_{i}(K, \omega)$ in the dual Brunn-Minkowski theory. Observe that $\widetilde{\mathrm{C}}_{i}\left(K, S^{n-1}\right)=\widetilde{\mathrm{W}}_{n-i}(K)$. As the area measure (cf. (1.5)), the dual curvature measures may also be considered as differentials of the dual quermassintegrals, even in a stronger form (see [25, Section 4]). We want to point out that there are also dual area measures corresponding to the area measures in the classical theory (see [25]).

Huang, Lutwak, Yang and Zhang also gave an explicit integral representation of the dual curvature measures which allowed them to define more generally for $q \in \mathbb{R}$ the $q$ th dual curvature measure of a convex body $K \in \mathcal{K}_{o}^{n}$ as [25, Def. 3.2]

$$
\widetilde{\mathrm{C}}_{q}(K, \eta)=\frac{1}{n} \int_{\alpha_{K}^{*}(\eta)} \rho_{K}(\boldsymbol{u})^{q} \mathrm{~d} \mathcal{H}^{n-1}(\boldsymbol{u}) .
$$

Here $\alpha_{K}^{*}(\eta)$ denotes the set of directions $\boldsymbol{u} \in S^{n-1}$, such that the boundary point $\rho_{K}(\boldsymbol{u}) \boldsymbol{u}$ belongs to $\nu_{K}^{-1}(\eta)$. The Minkowski-Christoffel problem may be considered as a charcterization problem of the differentials of quermassintegrals. Hence, the analog to the Minkowski-Christoffel problem in the dual Brunn-Minkowski theory is (cf. [25, Sect. 5]) 
The dual Minkowski problem. Given a finite Borel measure $\mu$ on $S^{n-1}$ and $q \in \mathbb{R}$. Find necessary and sufficient conditions for the existence of a convex body $K \in \mathcal{K}_{o}^{n}$ such that $\widetilde{\mathrm{C}}_{q}(K, \cdot)=\mu$.

An amazing feature of these dual curvature measures is that they also link two other well-known fundamental geometric measures of a convex body (cf. [25, Lemma 3.8]): when $q=0$ the dual curvature measure $\widetilde{\mathrm{C}}_{0}(K, \cdot)$ is up to a factor of $n$-Aleksandrov's integral curvature of the polar body of $K$ and for $q=n$ the dual curvature measure coincides with the cone-volume measure of $K$ given by

$$
\widetilde{\mathrm{C}}_{n}(K, \eta)=V_{K}(\eta)=\frac{1}{n} \int_{\nu_{K}^{-1}(\eta)}\left\langle\boldsymbol{u}, \nu_{K}(\boldsymbol{u})\right\rangle \mathrm{d} \mathcal{H}^{n-1}(\boldsymbol{u}) .
$$

Similarly to the Minkowski problem, solving the dual Minkowski problem is equivalent to solving a Monge-Ampère type partial differential equation if the measure $\mu$ has a density function $g: S^{n-1} \rightarrow \mathbb{R}$. In particular, if $q \in(0, n]$, then the dual Minkowski problem amounts to solving the MongeAmpère equation

$$
\frac{1}{n} h(\boldsymbol{x})|\nabla h(\boldsymbol{x})+h(\boldsymbol{x}) \boldsymbol{x}|^{q-n} \operatorname{det}\left[h_{i j}(\boldsymbol{x})+\delta_{i j} h(\boldsymbol{x})\right]=g(\boldsymbol{x}),
$$

where $\left[h_{i j}(\boldsymbol{x})\right]$ is the Hessian matrix of the (unknown) support function $h$ with respect to an orthonormal frame on $S^{n-1}$, and $\delta_{i j}$ is the Kronecker delta.

If $\frac{1}{n} h(\boldsymbol{x})|\nabla h(\boldsymbol{x})+h(\boldsymbol{x}) \boldsymbol{x}|^{q-n}$ were omitted in (1.7), then (1.7) would become the partial differential equation of the classical Minkowski problem, see, e.g., [9, 10, 38. If only the factor $|\nabla h(\boldsymbol{x})+h(\boldsymbol{x}) \boldsymbol{x}|^{q-n}$ were omitted, then equation (1.7) would become the partial differential equation associated with the cone volume measure, the so-called logarithmic Minkowski problem (see, e.g., [7, 11]). Due to the gradient component in (1.7) if $q \in(0, n)$, the dual Minkowski problem is signicantly more challenging than the classical Minkowski problem and logarithmic Minkowski problem.

The cone-volume measure for convex bodies has been studied extensively over the last few years in many different contexts, see, e.g., [2, 3, 6, 7, 8, 17, 19, 21, 25, 27, 28, 32, 33, 34, 35, 36, 37, 39, 43, 45, 46]. One very important property of the cone-volume measure - and which makes it so useful - is its $\mathrm{SL}(n)$-invariance, or simply called affine invariance. It is also the subject of the central logarithmic Minkowski problem which asks for sufficient and necessary conditions of a measure $\mu$ on $S^{n-1}$ to be the cone-volume measure of a convex body $K \in \mathcal{K}_{o}^{n}$. This is the $p=0$ limit case of the general $L_{p}$-Minkowski problem within the above mentioned $L_{p}$ Brunn-Minkowski theory for which we refer to [26, 31, 47] and the references within.

The discrete, planar, even case of the logarithmic Minkowski problem, i.e., with respect to origin-symmetric convex polygons, was completely solved by Stancu [41, 42, and later Zhu [45] as well as Böröczky, Hegedüs and Zhu [4] settled (in particular) the case when $K$ is a polytope whose outer normals are in general position.

In [7], Böröczky, Lutwak, Yang and Zhang gave a complete characterization of the cone-volume measure of origin-symmetric convex bodies among 
the even measures on the sphere. The key feature of such a measure is expressed via the following condition: A non-zero, finite Borel measure $\mu$ on the unit sphere satisfies the subspace concentration condition if

$$
\frac{\mu\left(S^{n-1} \cap L\right)}{\mu\left(S^{n-1}\right)} \leq \frac{\operatorname{dim} L}{n}
$$

for every proper subspace $L$ of $\mathbb{R}^{n}$, and whenever we have equality in (1.8) for some $L$, there is a subspace $L^{\prime}$ complementary to $L$, such that $\mu$ is concentrated on $S^{n-1} \cap\left(L \cup L^{\prime}\right)$.

Apart from the uniqueness aspect of the Minkowski problem, the symmetric case of the logarithmic Minkowski problem is settled.

Theorem 1.1 ([7]). A non-zero, finite, even Borel measure $\mu$ on $S^{n-1}$ is the cone-volume measure of $K \in \mathcal{K}_{e}^{n}$ if and only if $\mu$ satisfies the subspace concentration condition.

An extension of the validity of inequality 1.8 to centered bodies, i.e., bodies whose center of mass is at the origin, was given in the discrete case by Henk and Linke [23], and in the general setting by Böröczky and Henk [5].

A generalization (up to the equality case) of the sufficiency part of Theorem 1.1 to the $q$ th dual curvature measure for $q \in(0, n]$ was given by Huang, Lutwak, Yang and Zhang. For clarity, we separate their main result into the next two theorems.

Theorem 1.2 ([25, Theorem 6.6]). If $q \in(0,1]$, then an even finite Borel measure $\mu$ on $S^{n-1}$ is a qth dual curvature measure if and only if $\mu$ is not concentrated on any great subsphere.

Theorem 1.3 ([25, Theorem 6.6]). Let $q \in(1, n]$ and let $\mu$ be a non-zero, finite, even Borel measure on $S^{n-1}$ satisfying the subsapce mass inequality

$$
\frac{\mu\left(S^{n-1} \cap L\right)}{\mu\left(S^{n-1}\right)}<1-\frac{q-1}{q} \frac{n-\operatorname{dim} L}{n-1}
$$

for every proper subspace $L$ of $\mathbb{R}^{n}$. Then there exists an o-symmetric convex body $K \in \mathcal{K}_{e}^{n}$ with $\widetilde{\mathrm{C}}_{q}(K, \cdot)=\mu$.

In particular, it is highly desirable to understand how close 1.9$)$ is to characterize $q$ th dual curvature measures. Observe that for $q=n$ the inequality (1.9) becomes essentially (1.8).

Our main result treats the necessity of a subspace concentration bound on dual curvature measures.

Theorem 1.4. Let $K \in \mathcal{K}_{e}^{n}, q \in[1, n]$ and let $L \subset \mathbb{R}^{n}$ be a proper subspace. Then we have

$$
\frac{\widetilde{\mathrm{C}}_{q}\left(K, S^{n-1} \cap L\right)}{\widetilde{\mathrm{C}}_{q}\left(K, S^{n-1}\right)} \leq \min \left\{\frac{\operatorname{dim} L}{q}, 1\right\},
$$

and equality holds in 1.10 if and only if $q=n$ and $\widetilde{\mathrm{C}}_{n}(K, \cdot)$, i.e., the conevolume measure of $K$, satisfies the subspace concentration condition (1.8).

In particular, for $q<n$ we always have strict inequality in $(1.10)$, but this is also optimal. 
Proposition 1.5. Let $0<q<n$ and $k \in\{1, \ldots, n-1\}$. There exists a sequence of convex bodies $K_{l} \in \mathcal{K}_{e}^{n}, l \in \mathbb{N}$, and a $k$-dimensional subspace $L \subset \mathbb{R}^{n}$ such that

$$
\lim _{l \rightarrow \infty} \frac{\widetilde{\mathrm{C}}_{q}\left(K_{l}, S^{n-1} \cap L\right)}{\widetilde{\mathrm{C}}_{q}\left(K_{l}, S^{n-1}\right)}= \begin{cases}\frac{k}{q} & , k \leq q, \\ 1 & , k \geq q .\end{cases}
$$

We observe that if $q \in[1, n]$ and $\operatorname{dim} L=1$ for a linear subspace $L$ then $1-\frac{q-1}{q} \frac{n-\operatorname{dim} L}{n-1}=\frac{\operatorname{dim} L}{q}$. Therefore Theorems 1.3 and 1.4 complete the characterization of the $q$ th dual curvature measures if $n=2$.

Corollary 1.6. If $q \in[1,2)$, then an even finite Borel measure $\mu$ on $S^{1}$ is a qth dual curvature measure if and only if

$$
\frac{\mu\left(S^{1} \cap L\right)}{\mu\left(S^{1}\right)}<\frac{1}{q}
$$

for every one-dimensional subspace $L$ of $\mathbb{R}^{2}$.

We remark that the dual Minkowski problem is far easier to handle for the special case where the measure $\mu$ has a positive continuous density, (where subspace concentration is trivially satisfied). The singular general case for measures is substantially more delicate, which involves measure concentration and requires far more powerful techniques to solve.

The paper is organized as follows. First we will briefly recall some basic facts about convex bodies needed in our investigations in Section 2. In Section 3 we will prove a lemma in the spirit of the celebrated Brunn-Minkowski theorem, which is one of the main ingredients for the proof of Theorem 1.4 given in Section 4. Finally, in Section 5 we will prove Proposition 1.5.

\section{PRELIMINARIES}

We recommend the books by Gardner [15], Gruber [20] and Schneider [40] as excellent references on convex geometry.

For a given convex body $K \in \mathcal{K}^{n}$ the support function $h_{K}: \mathbb{R}^{n} \rightarrow \mathbb{R}$ is defined by

$$
h_{K}(\boldsymbol{x})=\max _{\boldsymbol{y} \in K}\langle\boldsymbol{x}, \boldsymbol{y}\rangle .
$$

A boundary point $\boldsymbol{x} \in \partial K$ is said to have a (not necessarily unique) unit outer normal vector $\boldsymbol{u} \in S^{n-1}$ if $\langle\boldsymbol{x}, \boldsymbol{u}\rangle=h_{K}(\boldsymbol{u})$. The corresponding supporting hyperplane $\left\{\boldsymbol{x} \in \mathbb{R}^{n}:\langle\boldsymbol{x}, \boldsymbol{u}\rangle=h_{K}(\boldsymbol{u})\right\}$ will be denoted by $H_{K}(\boldsymbol{u})$. For $K \in \mathcal{K}_{o}^{n}$ the radial function $\rho_{K}: \mathbb{R}^{n} \backslash\{\mathbf{0}\} \rightarrow \mathbb{R}$ is given by

$$
\rho_{K}(\boldsymbol{x})=\max \{\rho>0: \rho \boldsymbol{x} \in K\} .
$$

Note, that the support function and the radial function are homogeneous of degrees 1 and -1 , respectively, i.e.,

$$
h_{K}(\lambda \boldsymbol{x})=\lambda h_{K}(\boldsymbol{x}) \text { and } \rho_{K}(\lambda \boldsymbol{x})=\lambda^{-1} \rho_{K}(\boldsymbol{x}),
$$

for $\lambda>0$. We define the reverse radial Gauss image of $\eta \subseteq S^{n-1}$ with respect to a convex body $K \in \mathcal{K}_{o}^{n}$ by

$$
\alpha_{K}^{*}(\eta)=\left\{\boldsymbol{u} \in S^{n-1}: \rho_{K}(\boldsymbol{u}) \boldsymbol{u} \in H_{K}(\boldsymbol{v}) \text { for a } \boldsymbol{v} \in \eta\right\} .
$$


If $\eta$ is a Borel set, then $\alpha_{K}^{*}(\eta)$ is $\mathcal{H}^{n-1}$-measurable (see [40, Lemma 2.2.11.]) and so the $q$ th dual curvature measure given in (1.6) is well defined. We will need the following identity.

Lemma 2.1. Suppose $K \in \mathcal{K}_{o}^{n}, q>0$ and let $\eta \subseteq S^{n-1}$ be a Borel set. Then

$$
\widetilde{\mathrm{C}}_{q}(K, \eta)=\frac{q}{n} \int_{\boldsymbol{x} \in K, \boldsymbol{x} /|\boldsymbol{x}| \in \alpha_{K}^{*}(\eta)}|\boldsymbol{x}|^{q-n} \mathrm{~d} \mathcal{H}^{n}(\boldsymbol{x}) .
$$

Proof. By using spherical coordinates and 1.6

$$
\begin{aligned}
\frac{q}{n} \int_{\boldsymbol{x} \in K, \boldsymbol{x} /|\boldsymbol{x}| \in \alpha_{K}^{*}(\eta)}|\boldsymbol{x}|^{q-n} \mathrm{~d} \mathcal{H}^{n}(\boldsymbol{x}) \\
=\frac{1}{n} \int_{\alpha_{K}^{*}(\eta)}\left(\int_{0}^{\rho_{K}(\boldsymbol{u})} q r^{n-1} r^{q-n} \mathrm{~d} r\right) \mathrm{d} \mathcal{H}^{n-1}(\boldsymbol{u}) \\
=\frac{1}{n} \int_{\alpha_{K}^{*}(\eta)} \rho_{K}(\boldsymbol{u})^{q} \mathrm{~d} \mathcal{H}^{n-1}(\boldsymbol{u})=\widetilde{\mathrm{C}}_{q}(K, \eta) .
\end{aligned}
$$

Let $L$ be a linear subspace of $\mathbb{R}^{n}$. We write $K \mid L$ to denote the image of the orthogonal projection of $K$ onto $L$ and $L^{\perp}$ for the subspace orthogonal to $L$.

As usual, for two subsets $A, B \subseteq \mathbb{R}^{n}$ and reals $\alpha, \beta \geq 0$ the Minkowski combination is defined by

$$
\alpha A+\beta B=\{\alpha \boldsymbol{a}+\beta \boldsymbol{b}: \boldsymbol{a} \in A, \boldsymbol{b} \in B\} .
$$

By the well-known Brunn-Minkowski inequality we know that the $n$-th root of the volume of the Minkowski combination is a concave function. More precisely, for two convex bodies $K_{0}, K_{1} \subset \mathbb{R}^{n}$ and for $\lambda \in[0,1]$ we have

$$
\operatorname{vol}_{n}\left((1-\lambda) K_{0}+\lambda K_{1}\right)^{1 / n} \geq(1-\lambda) \operatorname{vol}_{n}\left(K_{0}\right)^{1 / n}+\lambda \operatorname{vol}_{n}\left(K_{1}\right)^{1 / n},
$$

where $\operatorname{vol}_{n}(\cdot)=\mathcal{H}^{n}(\cdot)$ denotes the $n$-dimensional Hausdorff measure. We have equality in (2.1) for some $0<\lambda<1$ if and only if $K_{0}$ and $K_{1}$ lie in parallel hyperplanes or they are homothetic, i.e., there exist a $t \in \mathbb{R}^{n}$ and $\mu \geq 0$ such that $K_{1}=\boldsymbol{t}+\mu K_{0}$ (see, e.g., [14], [40, Sect. 6.1]).

\section{INTEGRALS OF EVEN UNIMODAL FUNCTIONS}

A function $f$ on the real line $\mathbb{R}$ is called unimodal if there is a number $m \in \mathbb{R}$, such that $f$ is an increasing function on $(-\infty, m)$ and decreasing on $(m, \infty)$. The notion of unimodal functions can be extended to higher dimensional spaces in the following way.

The superlevel sets of a function $f: \mathbb{R}^{n} \rightarrow \mathbb{R}$ are given by $L_{f}^{+}(\alpha)=\{\boldsymbol{x} \in$ $\left.\mathbb{R}^{n}: f(\boldsymbol{x}) \geq \alpha\right\}, \alpha \in \mathbb{R}$. We say that $f$ is unimodal if every superlevel set of $f$ is closed and convex. It was shown by Anderson [1] that the integral of 
an even unimodal function over translates of a symmetric convex region is maximal if the center of symmetry is moved to the origin. His proof relies only on the Brunn-Minkowski theorem. Here we generalize this approach to integrals over a convex combination of a convex body $K$ and its reflection $-K$.

Lemma 3.1. Let $f: \mathbb{R}^{n} \rightarrow \mathbb{R}_{\geq 0} \cup\{\infty\}$ be a unimodal function, such that $f(\boldsymbol{x})=f(-\boldsymbol{x})$ for every $\boldsymbol{x} \in \mathbb{R}^{n}$ and let $f$ be integrable on compact, convex sets. Let $K \subset \mathbb{R}^{n}$ be a compact, convex set with $\operatorname{dim} K=k$. Then for $\lambda \in[0,1]$

$$
\int_{\lambda K+(1-\lambda)(-K)} f(\boldsymbol{x}) \mathrm{d} \mathcal{H}^{k}(\boldsymbol{x}) \geq \int_{K} f(\boldsymbol{x}) \mathrm{d} \mathcal{H}^{k}(\boldsymbol{x}) .
$$

Moreover, equality holds if and only if for every $\alpha>0$

$$
\operatorname{vol}_{k}\left([\lambda K+(1-\lambda)(-K)] \cap L_{f}^{+}(\alpha)\right)=\operatorname{vol}_{k}\left(K \cap L_{f}^{+}(\alpha)\right) .
$$

Proof. Let $K_{\lambda}=\lambda K+(1-\lambda)(-K)$. By the convexity of $L_{f}^{+}(\alpha)$ we have for every $\alpha \in \mathbb{R}$

$$
K_{\lambda} \cap L_{f}^{+}(\alpha) \supseteq \lambda\left(K \cap L_{f}^{+}(\alpha)\right)+(1-\lambda)\left((-K) \cap L_{f}^{+}(\alpha)\right) .
$$

The Brunn-Minkowski inequality (2.1) applied to the set on right hand side of $(3.2)$ gives

$$
\begin{aligned}
\operatorname{vol}_{k}\left(K_{\lambda}\right. & \left.\cap L_{f}^{+}(\alpha)\right) \\
& \geq \operatorname{vol}_{k}\left(\lambda\left(K \cap L_{f}^{+}(\alpha)\right)+(1-\lambda)\left((-K) \cap L_{f}^{+}(\alpha)\right)\right) \\
& \geq\left(\lambda \operatorname{vol}_{k}\left(K \cap L_{f}^{+}(\alpha)\right)^{1 / k}+(1-\lambda) \operatorname{vol}_{k}\left((-K) \cap L_{f}^{+}(\alpha)\right)^{1 / k}\right)^{k} .
\end{aligned}
$$

Since $f$ is even, the superlevel sets $L_{f}^{+}(\alpha)$ are symmetric. Hence, $\operatorname{vol}_{k}(K \cap$ $\left.L_{f}^{+}(\alpha)\right)=\operatorname{vol}_{k}\left((-K) \cap L_{f}^{+}(\alpha)\right)$ and so

$$
\operatorname{vol}_{k}\left(K_{\lambda} \cap L_{f}^{+}(\alpha)\right) \geq \operatorname{vol}_{k}\left(K \cap L_{f}^{+}(\alpha)\right)
$$

for every $\alpha \in \mathbb{R}$. Fubini's theorem yields

$$
\begin{aligned}
\int_{K_{\lambda}} f(x) \mathrm{d} \mathcal{H}^{k}(x) & =\int_{0}^{\infty} \operatorname{vol}_{k}\left(K_{\lambda} \cap L_{f}^{+}(\alpha)\right) \mathrm{d} \alpha \\
& \geq \int_{0}^{\infty} \operatorname{vol}_{k}\left(K \cap L_{f}^{+}(\alpha)\right) \mathrm{d} \alpha \\
& =\int_{K} f(x) \mathrm{d} \mathcal{H}^{k}(x) .
\end{aligned}
$$

Suppose we have equality in 3.1 . Since $\operatorname{vol}_{k}\left(K_{\lambda} \cap L_{f}^{+}(\alpha)\right)$ is continuous on the left with respect to $\alpha$ for every $\lambda \in[0,1]$ we find that

$$
\operatorname{vol}_{k}\left(K_{\lambda} \cap L_{f}^{+}(\alpha)\right)=\operatorname{vol}_{k}\left(K \cap L_{f}^{+}(\alpha)\right)
$$

for every $\alpha>0$. 


\section{Proof of Theorem 1.4}

Now we are ready to give the proof of Theorem 1.4. We use Fubini's theorem to decompose the dual curvature measure into integrals over hyperplane sections. Lemma 3.1 will provide a critical estimate for these integrals.

Proof of Theorem 1.4. In order to prove the inequality $(1.10)$ we may certainly assume $q>\operatorname{dim} L=k$. For $\boldsymbol{y} \in K \mid L$ denote $\overline{\boldsymbol{y}}=\rho_{K \mid L}(\boldsymbol{y}) \boldsymbol{y}$,

$$
\begin{aligned}
F_{\boldsymbol{y}} & =\operatorname{conv}\left\{\mathbf{0}, K \cap\left(\overline{\boldsymbol{y}}+L^{\perp}\right)\right\} \text { and } \\
M_{\boldsymbol{y}} & =\operatorname{conv}\left\{K \cap L^{\perp}, K \cap\left(\overline{\boldsymbol{y}}+L^{\perp}\right)\right\} .
\end{aligned}
$$

Observe that $M_{\boldsymbol{y}} \cap\left(\overline{\boldsymbol{y}}+L^{\perp}\right)=F_{\boldsymbol{y}} \cap\left(\overline{\boldsymbol{y}}+L^{\perp}\right)=K \cap\left(\overline{\boldsymbol{y}}+L^{\perp}\right)$. By Lemma 2.1 Fubini's theorem and the fact that $M_{\boldsymbol{y}} \cap\left(\boldsymbol{y}+L^{\perp}\right) \subseteq K \cap\left(\boldsymbol{y}+L^{\perp}\right)$ we may write

$$
\begin{aligned}
\widetilde{\mathrm{C}}_{q}\left(K, S^{n-1}\right) & =\frac{q}{n} \int_{K \mid L}\left(\int_{K \cap\left(\boldsymbol{y}+L^{\perp}\right)}|\boldsymbol{z}|^{q-n} \mathrm{~d} \mathcal{H}^{n-k}(\boldsymbol{z})\right) \mathrm{d} \mathcal{H}^{k}(\boldsymbol{y}) \\
& \geq \frac{q}{n} \int_{K \mid L}\left(\int_{\left.M_{\boldsymbol{y} \cap\left(\boldsymbol{y}+L^{\perp}\right)}|\boldsymbol{z}|^{q-n} \mathrm{~d} \mathcal{H}^{n-k}(\boldsymbol{z})\right) \mathrm{d} \mathcal{H}^{k}(\boldsymbol{y}) .}\right.
\end{aligned}
$$

In order to estimate the inner integral let $\boldsymbol{y} \in K \mid L, \boldsymbol{y} \neq \mathbf{0}$, and for abbreviation we set $\lambda=\rho_{K \mid L}(\boldsymbol{y})^{-1} \leq 1$. Then by the symmetry of $K$ we find

$$
\begin{aligned}
M_{\boldsymbol{y}} \cap\left(\boldsymbol{y}+L^{\perp}\right) \supseteq & \lambda\left(K \cap\left(\overline{\boldsymbol{y}}+L^{\perp}\right)\right)+(1-\lambda)\left(K \cap L^{\perp}\right) \\
\supseteq & \lambda\left(K \cap\left(\overline{\boldsymbol{y}}+L^{\perp}\right)\right) \\
& +(1-\lambda)\left(\frac{1}{2}\left(K \cap\left(\overline{\boldsymbol{y}}+L^{\perp}\right)\right)+\frac{1}{2}\left(-\left(K \cap\left(\overline{\boldsymbol{y}}+L^{\perp}\right)\right)\right)\right) \\
= & \frac{1+\lambda}{2}\left(K \cap\left(\overline{\boldsymbol{y}}+L^{\perp}\right)\right)+\frac{1-\lambda}{2}\left(-\left(K \cap\left(\overline{\boldsymbol{y}}+L^{\perp}\right)\right)\right) .
\end{aligned}
$$

Hence the set $M_{\boldsymbol{y}} \cap\left(\boldsymbol{y}+L^{\perp}\right)$ contains a convex combination of a set and its reflection at the origin. This allows us to apply Lemma 3.1 from which we get

$$
\int_{M_{\boldsymbol{y}} \cap\left(\boldsymbol{y}+L^{\perp}\right)}|\boldsymbol{z}|^{q-n} \mathrm{~d} \mathcal{H}^{n-k}(\boldsymbol{z}) \geq \int_{K \cap\left(\overline{\boldsymbol{y}}+L^{\perp}\right)}|\boldsymbol{z}|^{q-n} \mathrm{~d} \mathcal{H}^{n-k}(\boldsymbol{z})
$$

for every $\boldsymbol{y} \in K \mid L, \boldsymbol{y} \neq \mathbf{0}$. Together with 4.1) we obtain the lower bound

$$
\widetilde{\mathrm{C}}_{q}\left(K, S^{n-1}\right) \geq \frac{q}{n} \int_{K \mid L}\left(\int_{K \cap\left(\overline{\boldsymbol{y}}+L^{\perp}\right)}|\boldsymbol{z}|^{q-n} \mathrm{~d} \mathcal{H}^{n-k}(\boldsymbol{z})\right) \mathrm{d} \mathcal{H}^{k}(\boldsymbol{y}) .
$$

In order to evaluate $\widetilde{\mathrm{C}}_{q}\left(K, S^{n-1} \cap L\right)$ we note that for $\boldsymbol{x} \in K$ we have $\boldsymbol{x} /|\boldsymbol{x}| \in \alpha_{K}^{*}\left(S^{n-1} \cap L\right)$ if and only if the boundary point $\rho_{K}(\boldsymbol{x}) \boldsymbol{x}$ has an 
outer unit normal in $L$. Hence,

$$
\left\{\boldsymbol{x} \in K: \boldsymbol{x} /|\boldsymbol{x}| \in \alpha_{K}^{*}\left(S^{n-1} \cap L\right)\right\}=\operatorname{conv}\left\{\mathbf{0},\left(\partial(K \mid L)+L^{\perp}\right) \cap K\right\} \backslash\{\mathbf{0}\},
$$

and in view of Lemma 2.1 and Fubini's theorem we obtain

$$
\begin{aligned}
\widetilde{\mathrm{C}}_{q}(K, & \left.S^{n-1} \cap L\right) \\
& =\frac{q}{n} \int_{K \mid L}\left(\int_{\left.F_{\boldsymbol{y} \cap\left(\boldsymbol{y}+L^{\perp}\right)}|\boldsymbol{z}|^{q-n} \mathrm{~d} \mathcal{H}^{n-k}(\boldsymbol{z})\right) \mathrm{d} \mathcal{H}^{k}(\boldsymbol{y})} \int_{K \mid L}|\boldsymbol{z}|^{q-n} \mathrm{~d} \mathcal{H}^{n-k}(\boldsymbol{z})\right) \mathrm{d} \mathcal{H}^{k}(\boldsymbol{y}) \\
& =\frac{q}{n} \int_{\left(\rho_{K \mid L}(\boldsymbol{y})^{-1}\left(K \cap\left(\overline{\boldsymbol{y}}+L^{\perp}\right)\right)\right.}\left(\int_{K \cap\left(\overline{\boldsymbol{y}}+L^{\perp}\right)}|\boldsymbol{z}|^{q-n} \mathrm{~d} \mathcal{H}^{n-k}(\boldsymbol{z})\right) \mathrm{d} \mathcal{H}^{k}(\boldsymbol{y}) .
\end{aligned}
$$

The inner integral is independent of the length of $\boldsymbol{y} \in K \mid L$ and might be as well considered as the value $g(\boldsymbol{u})$ of a (measurable) function $g: S^{n-1} \cap L \rightarrow$ $\mathbb{R}_{\geq 0}$. By taking this into account and using spherical coordinates we obtain

$$
\begin{aligned}
\widetilde{\mathrm{C}}_{q}\left(K, S^{n-1} \cap L\right) \\
=\frac{q}{n} \int_{K \mid L} \rho_{K \mid L}(\boldsymbol{y})^{k-q} g(\boldsymbol{y} /|\boldsymbol{y}|) \mathrm{d} \mathcal{H}^{k}(\boldsymbol{y}) \\
=\frac{q}{n} \int_{S^{n-1} \cap L} g(\boldsymbol{u})\left(\int_{0}^{\rho_{K \mid L}(\boldsymbol{u})} \rho_{K \mid L}(r \boldsymbol{u})^{k-q} r^{k-1} \mathrm{~d} r\right) \mathrm{d} \mathcal{H}^{k-1}(\boldsymbol{u}) \\
=\frac{q}{n} \int_{S^{n-1} \cap L} g(\boldsymbol{u}) \rho_{K \mid L}(\boldsymbol{u})^{k-q}\left(\int_{0}^{\rho_{K \mid L}(\boldsymbol{u})} r^{q-1} \mathrm{~d} r\right) \mathrm{d} \mathcal{H}^{k-1}(\boldsymbol{u}) \\
=\frac{1}{n} \int_{S^{n-1} \cap L} g(\boldsymbol{u}) \rho_{K \mid L}(\boldsymbol{u})^{k} \mathrm{~d} \mathcal{H}^{k-1}(\boldsymbol{u}) .
\end{aligned}
$$

Applying the same transformation to the right hand side of 4.3 gives

$$
\begin{aligned}
\widetilde{\mathrm{C}}_{q}\left(K, S^{n-1}\right) & \geq \frac{q}{n} \int_{K \mid L} g(\boldsymbol{y} /|\boldsymbol{y}|) \mathrm{d} \mathcal{H}^{k}(\boldsymbol{y}) \\
& =\frac{q}{n} \int_{S^{n-1} \cap L} g(\boldsymbol{u})\left(\int_{0}^{\rho_{K \mid L}(\boldsymbol{u})} r^{k-1} \mathrm{~d} r\right) \mathrm{d} \mathcal{H}^{k-1}(\boldsymbol{u}) \\
& =\frac{q}{n} \frac{1}{k} \int_{S^{n-1} \cap L} g(\boldsymbol{u}) \rho_{K \mid L}(\boldsymbol{u})^{k} \mathrm{~d} \mathcal{H}^{k-1}(\boldsymbol{u}) .
\end{aligned}
$$


Combining (4.4) and (4.5) yields 1.10 in the case $k=\operatorname{dim} L \leq q$, i.e.,

$$
\frac{\widetilde{\mathrm{C}}_{q}\left(K, S^{n-1} \cap L\right)}{\widetilde{\mathrm{C}}_{q}\left(K, S^{n-1}\right)} \leq \frac{k}{q} .
$$

Now suppose that the dual curvature measure of $K$ satisfies the inequality (1.10) with equality with respect to a proper subspace $L$. Then we certainly have $\operatorname{dim} L<q$, since the curvature measure cannot be concentrated on a great hypersphere. Hence we must have equality in 4.2 for every $\boldsymbol{y} \in$ relint $(K \mid L)$.

Assume $q<n$. Then the superlevel sets $L_{f}^{+}(\alpha)$ of the function $f(\boldsymbol{z})=$ $|\boldsymbol{z}|^{q-n}, \boldsymbol{z} \in \mathbb{R}^{n}$, are balls. Hence, in view of the equality condition of Lemma 3.1. equality in (4.2) implies that $M_{\boldsymbol{y}} \cap\left(\boldsymbol{y}+L^{\perp}\right) \cap r B_{n}$ and $K \cap(\overline{\boldsymbol{y}}+$ $\left.L^{\perp}\right) \cap r \overline{B_{n}}$ have the same $(n-k)$-dimensional volume for every $r>0$. For sufficiently small $r$, however, the intersection of $K \cap\left(\overline{\boldsymbol{y}}+L^{\perp}\right) \subset \partial K$ with $r B_{n}$ is empty. Hence we must have $q=n$ and in this case we know by Theorem 1.1 that equality is attained if and only if the cone-volume measure of $K$ satisfies the subspace concentration condition as stated in Theorem 1.1 .

Remark 4.1. It is worth noting, that the proof of Theorem 1.4 only relies on the symmetry of the function $|\cdot|^{q-n}=\rho_{B_{n}}(\cdot)^{n-q}$, its homogeneity and the convexity of its unit ball. In fact, the ball $B_{n}$ can be replaced by any symmetric convex body $M \in \mathcal{K}_{e}^{n}$ in the sense that

$$
\begin{gathered}
\int_{\alpha_{K}^{*}\left(S^{n-1} \cap L\right)} \rho_{M}(\boldsymbol{u})^{n-q} \rho_{K}(\boldsymbol{u})^{q} \mathrm{~d} \mathcal{H}^{n-1}(\boldsymbol{u}) \leq \\
\frac{\operatorname{dim} L}{q} \int_{S^{n-1}} \rho_{M}(\boldsymbol{u})^{n-q} \rho_{K}(\boldsymbol{u})^{q} \mathrm{~d} \mathcal{H}^{n-1}(\boldsymbol{u}),
\end{gathered}
$$

where $L \subseteq \mathbb{R}^{n}$ is a subspace with $\operatorname{dim} L \leq q$. Observe, in this more general setting, Lemma 2.1 becomes

$$
\begin{aligned}
\int_{\alpha_{K}^{*}(\eta)} & \rho_{M}(\boldsymbol{u})^{n-q} \rho_{K}(\boldsymbol{u})^{q} \mathrm{~d} \mathcal{H}^{n-1}(\boldsymbol{u}) \\
= & \int_{\alpha_{K}^{*}(\eta)} \rho_{M}(\boldsymbol{u})^{n-q}\left(\int_{0}^{\rho_{K}(\boldsymbol{u})} q r^{n-1} r^{q-n} \mathrm{~d} r\right) \mathrm{d} \mathcal{H}^{n-1}(\boldsymbol{u}) \\
= & q \int_{\boldsymbol{x} \in K, \boldsymbol{x} /|\boldsymbol{x}| \in \alpha_{K}^{*}(\eta)} \rho_{M}(\boldsymbol{x})^{n-q} \mathrm{~d} \mathcal{H}^{n}(\boldsymbol{x}),
\end{aligned}
$$

and (4.6) can be proved along the same lines as Theorem 1.4 with $\rho_{B_{n}}(\cdot)$ replaced by $\rho_{M}(\cdot)$.

\section{Proof of Proposition 1.5}

Here we show that the bounds given in Theorem 1.4 are indeed tight for every choice of $q \in(0, n)$. To this end let $k \in \mathbb{N}$ with $0<k<n$ and for 
$r>0$ let $K_{r}$ be the cylinder

$$
K_{r}=\left(r B_{k}\right) \times B_{n-k}
$$

Let $L=\operatorname{lin}\left\{\boldsymbol{e}_{1}, \ldots, \boldsymbol{e}_{k}\right\}$ be the $k$-dimensional subspace generated by the first $k$ canonical unit vectors $\boldsymbol{e}_{i}$.

For $\boldsymbol{x} \in \mathbb{R}^{n}$ write $\boldsymbol{x}=\boldsymbol{x}_{1}+\boldsymbol{x}_{2}$, where $\boldsymbol{x}_{1} \in \mathbb{R}^{k} \times\{\mathbf{0}\}$ and $\boldsymbol{x}_{2} \in\{\mathbf{0}\} \times \mathbb{R}^{n-k}$. The supporting hyperplane of $K_{r}$ with respect to a unit vector $\boldsymbol{v} \in S^{n-1} \cap L$ is given by

$$
H_{K_{r}}(\boldsymbol{v})=\left\{\boldsymbol{x} \in \mathbb{R}^{n}:\left\langle\boldsymbol{v}, \boldsymbol{x}_{1}\right\rangle=r\right\}
$$

Hence the part of the boundary of $K_{r}$ covered by all these supporting hyperlanes is given by $r S^{k-1} \times B_{n-k}$. In view of Lemma 2.1 and Fubini's theorem we conclude

$$
\begin{aligned}
& \widetilde{\mathrm{C}}_{q}\left(K_{r}, S^{n-1} \cap L\right)= \\
& \quad \frac{q}{n} \int_{\boldsymbol{x}_{1} \in r B_{k}}\left(\int_{\substack{\boldsymbol{x}_{2} \in B_{n-k} \\
r\left|\boldsymbol{x}_{2}\right| \leq\left|\boldsymbol{x}_{1}\right|}}\left(\left|\boldsymbol{x}_{1}\right|^{2}+\left|\boldsymbol{x}_{2}\right|^{2}\right)^{\frac{q-n}{2}} \mathrm{~d} \mathcal{H}^{n-k}\left(\boldsymbol{x}_{2}\right)\right) \mathrm{d} \mathcal{H}^{k}\left(\boldsymbol{x}_{1}\right) .
\end{aligned}
$$

Denote the volume of $B_{n}$ by $\omega_{n}$. Recall, that the surface area of $B_{n}$ is given by $n \omega_{n}$ and for abbreviation we set

$$
c=c(q, k, n)=\frac{q}{n} k \omega_{k}(n-k) \omega_{n-k}
$$

Switching to the cylindrical coordinates

$$
\boldsymbol{x}_{1}=s \boldsymbol{u}, \quad s \geq 0, \boldsymbol{u} \in S^{k-1}, \quad \boldsymbol{x}_{2}=t \boldsymbol{v}, \quad t \geq 0, \boldsymbol{v} \in S^{n-k-1},
$$

transforms the right hand side of (5.1) to

$$
\begin{aligned}
\widetilde{\mathrm{C}}_{q}\left(K_{r}, S^{n-1} \cap L\right) & =c \int_{0}^{r} \int_{0}^{s / r} s^{k-1} t^{n-k-1}\left(s^{2}+t^{2}\right)^{\frac{q-n}{2}} \mathrm{~d} t \mathrm{~d} s \\
& =c \int_{0}^{r} \int_{0}^{1} s^{q-1} r^{k-n} t^{n-k-1}\left(1+r^{-2} t^{2}\right)^{\frac{q-n}{2}} \mathrm{~d} t \mathrm{~d} s \\
& =c r^{k} \int_{0}^{1} \int_{0}^{1} s^{q-1} t^{n-k-1}\left(r^{2}+t^{2}\right)^{\frac{q-n}{2}} \mathrm{~d} t \mathrm{~d} s .
\end{aligned}
$$


Analogously we obtain

$$
\begin{aligned}
& \widetilde{\mathrm{C}}_{q}\left(K_{r}, S^{n-1}\right) \\
& =\frac{q}{n} \int_{\boldsymbol{x}_{1} \in r B_{k}}\left(\int_{\boldsymbol{x}_{2} \in B_{n-k}}\left(\left|\boldsymbol{x}_{1}\right|^{2}+\left|\boldsymbol{x}_{2}\right|^{2}\right)^{\frac{q-n}{2}} \mathrm{~d} \mathcal{H}^{n-k}\left(\boldsymbol{x}_{2}\right)\right) \mathrm{d} \mathcal{H}^{k}\left(\boldsymbol{x}_{1}\right) \\
& =c \int_{0}^{r} \int_{0}^{1} s^{k-1} t^{n-k-1}\left(s^{2}+t^{2}\right)^{\frac{q-n}{2}} \mathrm{~d} t \mathrm{~d} s \\
& =c r^{k} \int_{0}^{1} \int_{0}^{1} s^{k-1} t^{n-k-1}\left(r^{2} s^{2}+t^{2}\right)^{\frac{q-n}{2}} \mathrm{~d} t \mathrm{~d} s .
\end{aligned}
$$

When $q>k$, the monotone convergence theorem gives

$$
\begin{aligned}
\lim _{r \rightarrow 0+} \int_{0}^{1} \int_{0}^{1} s^{q-1} t^{n-k-1}\left(r^{2}+t^{2}\right)^{\frac{q-n}{2}} \mathrm{~d} t \mathrm{~d} s \\
=\int_{0}^{1} s^{q-1} \mathrm{~d} s \cdot \int_{0}^{1} t^{q-k-1} \mathrm{~d} t=\frac{1}{q(q-k)}
\end{aligned}
$$

and

$$
\begin{aligned}
\lim _{r \rightarrow 0+} \int_{0}^{1} \int_{0}^{1} s^{k-1} t^{n-k-1}\left(r^{2} s^{2}+t^{2}\right)^{\frac{q-n}{2}} \mathrm{~d} t \mathrm{~d} s \\
=\int_{0}^{1} s^{k-1} \mathrm{~d} s \cdot \int_{0}^{1} t^{q-k-1} \mathrm{~d} t=\frac{1}{k(q-k)} .
\end{aligned}
$$

Hence, by (5.2) and 5.3 we get

$$
\lim _{r \rightarrow 0+} \frac{\widetilde{\mathrm{C}}_{q}\left(K_{r}, S^{n-1} \cap L\right)}{\widetilde{\mathrm{C}}_{q}\left(K_{r}, S^{n-1}\right)}=\frac{k}{q}
$$

Now suppose $q \leq k$. Rewrite $(5.3)$ as

$$
\widetilde{\mathrm{C}}_{q}\left(K_{r}, S^{n-1}\right)=c r^{k} \int_{0}^{1} \int_{0}^{1 / s} s^{q-1} t^{n-k-1}\left(r^{2}+t^{2}\right)^{\frac{q-n}{2}} \mathrm{~d} t \mathrm{~d} s,
$$

which in view of $(5.2)$ gives

$$
\begin{aligned}
\widetilde{\mathrm{C}}_{q}\left(K_{r}, S^{n-1} \cap L\right) & -\widetilde{\mathrm{C}}_{q}\left(K_{r}, S^{n-1}\right) \\
& =c r^{k} \int_{0}^{1} \int_{1}^{1 / s} s^{q-1} t^{n-k-1}\left(r^{2}+t^{2}\right)^{\frac{q-n}{2}} \mathrm{~d} t \mathrm{~d} s .
\end{aligned}
$$


Observe, that by the monotone convergence theorem

$$
\begin{aligned}
\lim _{r \rightarrow 0+} \int_{0}^{1} \int_{1}^{1 / s} s^{q-1} t^{n-k-1}\left(r^{2}+t^{2}\right)^{\frac{q-n}{2}} \mathrm{~d} t \mathrm{~d} s=\int_{0}^{1} \int_{1}^{1 / s} s^{q-1} t^{q-k-1} \mathrm{~d} t \mathrm{~d} s \\
= \begin{cases}\int_{0}^{1} s^{q-1} \frac{1-s^{k-q}}{k-q} \mathrm{~d} s, & \text { if } q<k, \\
\int_{0}^{1} s^{q-1}(-\log s) \mathrm{d} s, & \text { if } q=k, \\
& =\frac{1}{k q} .\end{cases}
\end{aligned}
$$

On the other hand, if $0<r<1$, then

$$
\begin{aligned}
\int_{0}^{1} \int_{0}^{1 / s} s^{q-1} t^{n-k-1}\left(r^{2}+t^{2}\right)^{\frac{q-n}{2}} \mathrm{~d} t \mathrm{~d} s \\
\quad \geq \int_{0}^{1} \int_{r}^{1} s^{q-1} t^{n-k-1}(r+t)^{q-n} \mathrm{~d} t \mathrm{~d} s \\
\geq \int_{0}^{1} \int_{r}^{1} s^{q-1} t^{n-k-1}(t+t)^{q-n} \mathrm{~d} t \mathrm{~d} s \\
= \begin{cases}\frac{2^{q-n}}{q} \frac{r^{q-k}-1}{k-q}, & \text { if } q<k, \\
\frac{2^{q-n}}{q}(-\log (r)), & \text { if } q=k,\end{cases}
\end{aligned}
$$

which is not bounded from above as a function in $r$. Hence, by (5.4), (5.5), (5.6), (5.7) we finally get

$$
\begin{aligned}
\lim _{r \rightarrow 0+} \frac{\widetilde{\mathrm{C}}_{q}\left(K_{r}, S^{n-1} \cap L\right)}{\widetilde{\mathrm{C}}_{q}\left(K_{r}, S^{n-1}\right)} \\
\quad=1-\lim _{r \rightarrow 0+} \frac{\int_{0}^{1} \int_{1}^{1 / s} s^{q-1} t^{n-k-1}\left(r^{2}+t^{2}\right)^{\frac{q-n}{2}} \mathrm{~d} t \mathrm{~d} s}{\int_{0}^{1 / s} \int_{0}^{1 / s} s^{q-1} t^{n-k-1}\left(r^{2}+t^{2}\right)^{\frac{q-n}{2}} \mathrm{~d} t \mathrm{~d} s} \\
\quad=1,
\end{aligned}
$$

which finishes the proof of Proposition 1.5 .

Acknowledgement. The authors would like to thank the referees for their very helpful comments and suggestions.

\section{REFERENCES}

[1] Theodore Wilbur Anderson. The integral of a symmetric unimodal function over a symmetric convex set and some probability inequalities. Proc. Amer. Math. Soc., 6:170-176, 1955. MR0069229, Zbl 0066.37402

[2] Franck Barthe, Olivier Guédon, Shahar Mendelson, and Assaf Naor. A probabilistic approach to the geometry of the $l_{p}^{n}$-ball. Ann. Probab., 33(2):480-513, 2005. MR2123199, Zbl 1071.60010 
[3] K. J. Böröczky and P. Hegedüs. The cone volume measure of antipodal points. Acta Math. Hungar., 146(2):449-465, 2015. MR3369507

[4] Károly J. Böröczky, Pál Hegedűs, and Guangxian Zhu. On the discrete logarithmic minkowski problem. International Mathematics Research Notices, 2015. MR3509941, Zbl 06572638

[5] Károly J. Böröczky and Martin Henk. Cone-volume measure of general centered convex bodies. Adv. Math., 286:703-721, 2016. MR3415694, Zbl 1334.52003

[6] Károly J. Böröczky, Erwin Lutwak, Deane Yang, and Gaoyong Zhang. The logBrunn-Minkowski inequality. Adv. Math., 231(3-4):1974-1997, 2012. MR2964630, Zbl 1258.52005

[7] Károly J. Böröczky, Erwin Lutwak, Deane Yang, and Gaoyong Zhang. The logarithmic Minkowski problem. J. Amer. Math. Soc., 26(3):831-852, 2013. MR3037788, Zbl 1272.52012

[8] Károly J. Böröczky, Erwin Lutwak, Deane Yang, and Gaoyong Zhang. Affine images of isotropic measures. J. Differential Geom., 99(3):407-442, 2015. MR3316972, Zbl 1325.28004

[9] Luis Caffarelli. Interior $W^{2, p}$-estimates for solutions of the Monge-Ampere equation. Ann. of Math., 131:135-150, 1990. MR1038360

[10] Shiu-Yuen Cheng, Shing-Tung Yau. On the regularity of the solution of the $n$ dimensional Minkowski problem. Commun. Pure Appl. Math., 29:495-516, 1976. MR0423267, Zbl 0363.53030

[11] Kai-Seng Chou, Xu-Jia Wang. The $L_{p}$-Minkowski problem and the Minkowski problem in centroaffine geometry. Adv. Math., 205:33-83, 2006. MR2254308, Zbl 1245.52001

[12] William J. Firey. p-means of convex bodies. Math. Scand., 10, 17-24, 1962. MR0141003, Zbl 0188.27303

[13] Richard J. Gardner. A positive answer to the Busemann-Petty problem in three dimensions. Ann. of Math. (2), 140:435-447, 1994. MR1298719, Zbl 0826.52010

[14] Richard J. Gardner. The Brunn-Minkowski inequality. Bull. Amer. Math. Soc. (N.S.), 39(3):355-405, 2002. MR1898210, Zbl 1019.26008

[15] Richard J. Gardner. Geometric tomography, volume 58 of Encyclopedia of Mathematics and its Applications. Cambridge University Press, Cambridge, second edition, 2006. MR2251886, Zbl 1102.52002

[16] Richard J. Gardner, Daniel Hug, and Wolfgang Weil. Operations between sets in geometry. J. Eur. Math. Soc. (JEMS), 15(6): 2297-2352, 2013. MR3120744, Zbl 1282.52006

[17] Richard J. Gardner, Daniel Hug, and Wolfgang Weil. The Orlicz-Brunn-Minkowski theory: a general framework, additions, and inequalities. J. Differential Geom., 97(3):427-476, 2014. MR3263511, Zbl 1303.52002

[18] Richard J. Gardner, Alexander Koldobsky, and Thomas Schlumprecht. An analytic solution to the Busemann-Petty problem on sections of convex bodies. Ann. of Math. (2), 149:691-703, 1999. MR1689343, Zbl 0937.52003

[19] Michail Gromov and Vitali D. Milman. Generalization of the spherical isoperimetric inequality to uniformly convex Banach spaces. Compositio Math., 62(3):263-282, 1987. MR0901393, Zbl 0623.46007

[20] Peter M. Gruber. Convex and discrete geometry, volume 336 of Grundlehren der Mathematischen Wissenschaften [Fundamental Principles of Mathematical Sciences]. Springer, Berlin, 2007. MR2335496, Zbl 1139.52001

[21] Christoph Haberl and Lukas Parapatits. The centro-affine Hadwiger theorem. J. Amer. Math. Soc., 27(3):685-705, 2014. MR3194492, Zbl 1319.52006

[22] Binwu He, Gangsong Leng, and Kanghai Li. Projection problems for symmetric polytopes. Adv. Math., 207(1):73-90, 2006. MR2264066, Zbl 1111.52012c

[23] Martin Henk and Eva Linke. Cone-volume measures of polytopes. Adv. Math., 253:5062, 2014. MR3148545, Zbl 1308.52007

[24] Martin Henk, Achill Schürmann, and Jörg M. Wills. Ehrhart polynomials and successive minima. Mathematika, 52(1-2):1-16 (2006), 2005. MR2261838, Zbl 1105.52011 
[25] Yong Huang, Erwin Lutwak, Deane Yang, and Gaoyong Zhang. Geometric measures in the dual brunn-minkowski theory and their associated minkowski problems. Acta Mathematica, 2016. in press.

[26] Daniel Hug, Erwin Lutwak, Deane Yang, and Gaoyong Zhang. On the $L_{p}$ Minkowski problem for polytopes. Discrete Comput. Geom., 33(4):699-715, 2005. MR2132298, Zbl 1078.52008

[27] Monika Ludwig. General affine surface areas. Adv. Math., 224(6):2346-2360, 2010. MR2652209, Zbl 1198.52004

[28] Monika Ludwig and Matthias Reitzner. A classification of $\operatorname{SL}(n)$ invariant valuations. Ann. of Math. (2), 172(2):1219-1267, 2010. MR2680490, Zbl 1223.52007

[29] Erwin Lutwak. Dual mixed volumes. Pacific J. Math., 58(2):531-538, 1975. MR0380631, Zbl 0273.52007

[30] Erwin Lutwak. Dual cross-sectional measures. Atti Accad. Naz. Lincei Rend. Cl. Sci. Fis. Mat. Natur. (8), 58(1):1-5, 1975. MR0415505, Zbl 0335.28007

[31] Erwin Lutwak. The Brunn-Minkowski-Firey theory. I. Mixed volumes and the Minkowski problem. J. Differential Geom., 38(1):131-150, 1993. MR1231704, Zbl 0788.52007

[32] Erwin Lutwak, Deane Yang, and Gaoyong Zhang. $L_{p}$ John ellipsoids. Proc. London Math. Soc. (3), 90(2):497-520, 2005. MR2142136, Zbl 1074.52005

[33] Erwin Lutwak, Deane Yang, and Gaoyong Zhang. Orlicz centroid bodies. J. Differential Geom., 84(2):365-387, 2010. MR2652465, Zbl 1206.49050

[34] Erwin Lutwak, Deane Yang, and Gaoyong Zhang. Orlicz projection bodies. Adv. Math., 223(1):220-242, 2010. MR2563216, Zbl 05643962

[35] Lei Ma. A new proof of the log-Brunn-Minkowski inequality. Geom. Dedicata, 177:7582, 2015. MR3370024, Zbl 06468782

[36] Assaf Naor. The surface measure and cone measure on the sphere of $l_{p}^{n}$. Trans. Amer. Math. Soc., 359(3):1045-1079 (electronic), 2007. MR2262841, Zbl 1109.60006

[37] Assaf Naor and Dan Romik. Projecting the surface measure of the sphere of $l_{p}^{n}$. Ann. Inst. H. Poincaré Probab. Statist., 39(2):241-261, 2003. MR1962135, Zbl 1012.60025

[38] Louis Nirenberg. The Weyl and Minkowski problems in differential geometry in the large. Comm. Pure Appl. Math., 6:337-394 1953. MR0058265, Zbl 0051.12402

[39] Grigoris Paouris and Elisabeth M. Werner. Relative entropy of cone measures and $L_{p}$ centroid bodies. Proc. Lond. Math. Soc. (3), 104(2):253-286, 2012. MR2880241, Zbl 1246.52008

[40] Rolf Schneider. Convex bodies: the Brunn-Minkowski theory, volume 44 of Encyclopedia of Mathematics and its Applications. Cambridge University Press, Cambridge, 1993, Second expanded edition, 2014. MR3155183, Zbl 1287.52001

[41] Alina Stancu. The discrete planar $L_{0}$-Minkowski problem. Adv. Math., 167(1):160174, 2002. MR1901250, Zbl 1005.52002

[42] Alina Stancu. On the number of solutions to the discrete two-dimensional $L_{0^{-}}$ Minkowski problem. Adv. Math., 180(1):290-323, 2003. MR2019226, Zbl 1054.52001

[43] Alina Stancu. Centro-affine invariants for smooth convex bodies. Int. Math. Res. Not. IMRN, (10):2289-2320, 2012. MR2923167, Zbl 1250.52009

[44] Gaoyong Zhang A positive solution to the Busemann-Petty problem in $\mathbf{R}^{4}$. Ann. of Math. (2), 149:535-543, 1999. MR1689339, Zbl 0937.52004

[45] Guangxian Zhu. The logarithmic Minkowski problem for polytopes. Adv. Math., 262:909-931, 2014. MR3228445, Zbl 1321.52015

[46] Guangxian Zhu. The centro-affine Minkowski problem for polytopes. J. Differential Geom., 101(1):159-174, 2015. MR3356071, Zbl 1331.53016

[47] Guangxian Zhu. The $L_{p}$ Minkowski problem for polytopes for $0<p<1$. J. Funct. Anal., 269(4):1070-1094, 2015. MR3352764, Zbl 1335.52023 
Alfréd Rényi Institute of Mathematics, Hungarian Academy of Sciences, ReÁltanoda u. 13-15., H-1053 Budapest, Hungary

E-mail address: carlos@renyi.hu

Technische Universität Berlin, Institut für Mathematik, Sekr. MA4-1, Strasse Des 17. Juni 136, D-10623 Berlin, Germany

E-mail address: henk@math.tu-berlin.de, pollehn@math.tu-berlin.de 